Review

\title{
Impact of Crocus satious L. on Metabolic Profile in Patients with Diabetes Mellitus or Metabolic Syndrome: A Systematic Review
}

\author{
Parthena Giannoulaki $^{1}$, Evangelia Kotzakioulafi ${ }^{2}{ }^{(D}$, Michail Chourdakis ${ }^{3}{ }^{(D)}$, \\ Apostolos Hatzitolios ${ }^{2}$ and Triantafyllos Didangelos ${ }^{2, *}$ \\ 1 Department of Nutrition and Dietetics, University General Hospital of Thessaloniki AHEPA, \\ 54621 Thessaloniki, Greece; nenagian@yahoo.com \\ 2 Diabetes Center, 1st Propeudetic Department of Internal Medicine, School of Health Sciences, Medical \\ School, Aristotle University of Thessaloniki, 54621 Thessaloniki, Greece; evelinakotzak@hotmail.com (E.K.); \\ chatzito@auth.gr (A.H.) \\ 3 Laboratory of Hygiene, Social \& Preventive Medicine and Medical Statistics, Department of Medicine, \\ School of Health Sciences, Aristotle University of Thessaloniki, 54124 Thessaloniki, Greece; \\ mhourd@gapps.auth.gr \\ * Correspondence: didang@med.auth.gr; Tel.: +30-694-486-3803
}

Received: 15 April 2020; Accepted: 13 May 2020; Published: 14 May 2020

\begin{abstract}
Background: Experimental studies demonstrated a positive effect of administration of Crocus sativus L. (saffron) and its bioactive ingredients on metabolic profile through their antioxidant capacity. Purpose: To determine if the use of saffron in humans is beneficial to patients with diabetes mellitus (DM) or metabolic syndrome (MS). Methods: This systematic review includes 14 randomized control trials that investigated the impact of saffron administration and its bioactive ingredient crocin on the metabolic profile of patients with DM, MS, prediabetes, and coronary artery disease. We documented the following clinical outcomes: fasting blood glucose (FBG), glycated haemoglobin (HbA1c), total cholesterol, low-density lipoprotein (LDL) cholesterol, high-density lipoprotein (HDL) cholesterol, triglycerides, systolic, and diastolic blood pressure. Results: Eight studies examined the efficacy of saffron in patients with DM, four with the metabolic syndrome, one with prediabetes and one with coronary artery disease. A favorable effect on FBG was observed. The results regarding blood lipids and blood pressure were inconclusive in the current review. Conclusions: According to the available limited evidence, saffron may have a favorable effect on FBG. Many of the studies in the reviewed literature are of poor quality, and more research is needed in this direction to confirm and establish the above findings.
\end{abstract}

Keywords: diabetes mellitus; Crocus sativus L.; saffron; crocin; picrocrocin; safranal; dyslipidemia; hyperglycemia

\section{Introduction}

Diabetes mellitus (DM) is a disorder of the metabolism of carbohydrates, proteins, and lipids, and its principal characteristic is hyperglycemia due to lower secretion or lack of insulin. Prolonged uncontrolled DM leads to certain microvascular complications such as nephropathy, neuropathy, retinopathy, and macrovascular complications, such as cardiovascular disease and stroke. Poor glycemic control is the main cause worldwide of end-stage chronic kidney disease, amputations, and blindness [1]. Due to DM's increasing prevalence globally, it has already become a major cause of cardiovascular morbidity and mortality. Moreover, costs for DM treatment and its complications are a substantial economic burden for many countries. Furthermore, patients with DM complications have a decreased 
quality of life and life expectancy [2,3].However, optimal control of plasma glucose and lipid concentrations can reduce the incidence of DM-related complications [4], but optimal metabolic control is difficult to achieve and maintain over time, especially in type $1 \mathrm{DM}$ patients.

Medical nutrition therapyis the cornerstone of the prevention and management of DM. Appropriate healthy eating habits, including low-carbohydrate, low glycemic load, and high-fiber diets with regular physical activity, and adequate sleep duration are associated with optimal glycemic control and achieving ideal body weight. Moreover, the favorable effects of the most popular glucose-lowering agents, dipeptidyl peptidase-4 (DPP-4) inhibitors, and sodium-glucose cotransporter 2 (SGLT-2) inhibitors are significantly affected by body mass index (BMI) and dietary patterns [5].

Although there are many treatment options for DM, the high cost of DM medication and its side effects have led researchers to investigate alternative treatment options. Among those is the use of saffron due to its high antioxidant capacity, which in many studies has been shown to exert a protective action against cell and tissue damage.

The plant Crocus sativus L. is a bulbous and perennial plant with red stigmas. Its red stigmas in dried form is the spice commonly known as saffron or crocus [6]. Saffron is produced mainly in Greece, Iran, and India. The plant extract contains potential pharmacological active ingredients, such: crocins (mono- and diglycosylic esters of dicarboxylic acids, crocetin), picrocrocin and safranal. The primary active ingredients are crocins (approximately $10 \%$ of the total content) [7]. High-quality saffron consists of approximately $30 \%$ crocins, $5-15 \%$ picrocrocin, and often $2.5 \%$ volatile compounds, one of which is safranal. Greek saffron, known as Greek red saffron, has the highest concentration of the above ingredients [8].

Experimental studies in animals have shown that saffron demonstrates antidiabetic and antioxidant properties. These studies showed that saffron and its bioactive components have a positive impact on hyperglycemia due to the improvement of fasting blood glucose (FBG) on serum insulin and $\mathrm{HbA1c}$ levels, advanced glycation end products (AGEs) production [9-14] and insulin sensitivity [15-17].

Moreover, in vitro and in vivo studies demonstrated the potential of saffron and its constituents in reducing the level of total serum cholesterol(t-chol), low-density lipoprotein cholesterol (LDL-c) and triglycerides (TG) and improving the levels of high-density cholesterol (HDL-c) and the ratio of LDL-c/HDL-c in healthy, diabetic and dyslipidemic animals [9,18-23].

In addition, it has been reported that the aqueous extract of saffron and its two compounds, crocin and safranal, can reduce mean arterial blood pressure in animals in a dose-dependent manner [24-27].

Research indicates that saffron and its constituents have a significant role in the inhibition and regression of atherosclerosis by preventing apoptosis in animal models [28-35] and improve adverse results from myocardial injury by significantly reducing the levels of lactate dehydrogenase (LDH), creatine kinase (CK), malondialdehyde(MDA)and increasing the level of superoxide dismutase(SOD) in rat myocardial ischemia model [36-42].

The balance between the removal and the production of cellular reactive oxygen species (ROS) is defined as the "redox state". There is no doubt that ROS are increased in the setting of DM. Many studies have tried to eliminate increased production with various agents (vitamins, antioxidants, etc.), but their results were inconclusive, possibly because we do not know the exact level of the removal of ROS. Some levels of ROS are needed for cell functionality. On the other hand, a dietary pattern rich in antioxidants, as is the Mediterranean dietary pattern, could provide a considerable reduction in cardiovascular risk and may be of particular benefit to subjects with diabetes mellitus [43].

Overall, a growing body of evidence has focused on the medicinal properties of saffron as an antidiabetic, hypolipidemic, anti-hypertensive, and cardioprotective agent in animals. However, previous work that examines the above properties in patients with DM and metabolic syndrome (MS) is limited. This review aimed to present and assess the results of relevant studies, regarding the impact of saffron and its bioactive components on the metabolic profile of patients with DM and the MS. Also, existing gaps in the available literature are discussed, and potential areas of future research are proposed. 


\section{Materials and Methods}

This systematic review was conducted following the guidelines of Preferred Reporting Items for Systematic Reviews and Meta-Analyses (PRISMA) (checklist is included as Supplementary Materials) and PRISMA flow diagram is presented in Figure 1.

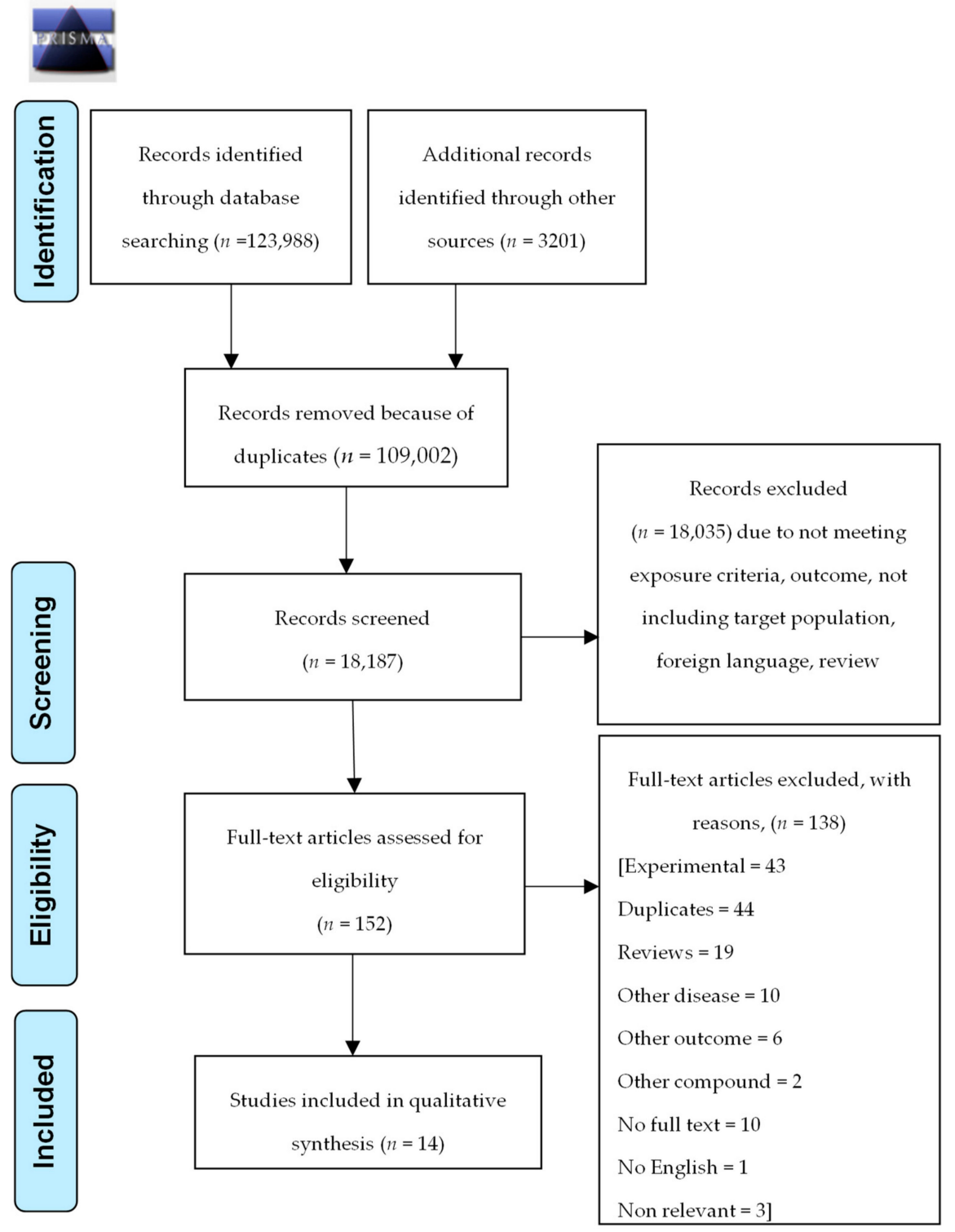

Figure 1. PRISMA flow diagram.

\subsection{Literature Search Strategy}

Two reviewers (PG and EK) independently performed an extensive literature search from July 2019 until September 2019 from the following databases: MEDLINE (via PubMed), Scopus (Science Direct), Cochrane Library Database of Systematic Reviews, Google Scholar and Clinicaltrials.gov. A final search was performed in January 2020 to identify any new publications. No filter was used during the search process. A combination of keywords and Boolean logic was used to search in all databases including "diabetes mellitus" or "antidiabetic" or "hyperglycemia" or "hypertension" or "metabolic syndrome" or "dyslipidemia" or "hyperlipidemia" or "hypoglycemia" or "atherosclerosis" or "macrovascular diabetic complications" or "microvascular diabetic complications" or "cardiovascular disease" or "myocardial injury" or "insulin sensitivity" or "insulin resistance" and "crocus sativus" or "crocin" or "picrocrocin" or "saffron" or "safranal". Also, an additional search was performed in PubMed using Medical Subject 
Headings (Mesh): "Diabetes Mellitus"[Mesh] AND ((((“Crocus"[Mesh]) OR “crocin” [Supplementary Concept]) OR "picrocrocin" [Supplementary Concept]) OR "safranal" [Supplementary Concept].

\subsection{Types of Studies and Eligibility Criteria}

Reviews, meta-analyses, experimental studies in vitro, and in vivo and clinical trials that were ongoing or had not published results yet were excluded from the search process. Additionally, any study for which full text was not retrieved or was not available in English language was also excluded. Furthermore, studies that examined different outcomes other than the metabolic profile or studied other diseases or other herbal compounds were excluded too. Only randomized controlled trials (RCTs) in human subjects with DM and MS were included. Studies included had biochemical metabolic markers, such as t-chol, HDL-c, LDL-c, TGlevels, FBG, HbA1c, waist circumference (WC), systolic and diastolic blood pressure (SBP and DBP) as an outcome.

\subsection{Data Collection and Extraction}

Abstracts of full texts were independently read by two reviewers (PG and EK) to assess their eligibility for this review and evaluated according to the eligibility criteria. Information and results that were of interest in each study were reported in a standardized manner. The extracted data from each study included the following characteristics: (I) citation, author, publication year and purpose; (II) inclusion and exclusion criteria; (III) type of intervention and type and amount of substance used; (IV) sample size; (V) baseline and after intervention metabolic variables; (VI) if dietary and physical activity assessments were used; (VII) reported conclusions; (VIII) funding sources and (IX) conflict of interest statement.

\subsection{Study Quality and Risk of Bias Assessment in Included Studies}

The quality of each study was independently assessed by two reviewers (PG and EK) using Cochrane Collaboration's tool for assessing the risk of bias in RCTs. Assessment of each study was entered into the software Review Manager 5.3. Critical assessment of several domains was performed, including selection bias, performance bias, detection bias, attrition bias, reporting bias, and other biases. Each assessment was characterized as either low or high or unclear risk. Two figures were generated by Review Manager to demonstrate all assessments of the included studies regarding risk of bias. There was no blinding of reviewers regarding study authors and journal. Consensus was reached for all studies included.

\subsection{Data Synthesis and Analysis}

Narrative synthesis and analysis of the data of each study was made. No meta-analysis was performed due to the high heterogeneity regarding study design and reported outcomes between included studies. Wherever there were two intervention groups in a study, the statistics of these two groups were combined in one, using the handbook Cochrane formula for combining two groups.

\section{Results}

\subsection{Characteristics of the Studies Included in the Review}

Fourteen studies met the eligibility criteria for this systematic review. All studies were randomized placebo-controlled trials, eight in patients with DM [44-51], four with MS [52-55], one with prediabetes [56], and one with coronary artery disease [57]. All of them had at least one intervention arm with oral administration of saffron extract or crocin and a placebo arm. Reported outcomes were changes in metabolic variables such as FBG, HbA1c, t-chol, HDL-c, LDL-c, TG, WC, SBP, and DBP (Table 1). 
Table 1. Summary of study characteristics.

\begin{tabular}{|c|c|c|c|c|c|c|c|c|c|c|}
\hline Study (Year) & Sample & $\begin{array}{l}\text { Gender } \\
\text { (Males) }\end{array}$ & Age (Years) & Study Design & Condition & Intervention/Groups & Duration & Outcome & $\begin{array}{c}\text { Assessment } \\
\text { of Diet }\end{array}$ & $\begin{array}{c}\text { Assessmen } \\
\text { of Physical } \\
\text { Activity }\end{array}$ \\
\hline $\begin{array}{l}\text { Abedimanesh } \\
2017[57]\end{array}$ & 75 & $14 / 13 / 12$ & $\begin{array}{l}56.04 \pm 7.55 \\
53.36 \pm 5.94 \\
56.32 \pm 5.91\end{array}$ & $\begin{array}{l}\text { Randomized } \\
\text { Double blind } \\
\text { placebo controlled }\end{array}$ & $\begin{array}{l}\text { Coronary } \\
\text { artery } \\
\text { disease }(17 \% \\
\text { DM) }\end{array}$ & $\begin{array}{l}\text { Saffron aqueous extract } \\
\text { ( } 30 \mathrm{mg}) \text { vs. } \\
\text { Crocin }(30 \mathrm{mg}) \text { vs. } \\
\text { Placebo }\end{array}$ & 8 weeks & $\begin{array}{c}\text { FBG, t-chol, } \\
\text { HDL-c, } \\
\text { LDL-c, TG, WC }\end{array}$ & Yes & No \\
\hline $\begin{array}{c}\text { Azimi } 2014 \\
{[44]}\end{array}$ & 208 & $17 / 16 / 15 / 16 / 15$ & $54.33 \pm 0.5$ & $\begin{array}{c}\text { Parallel } \\
\text { Randomized } \\
\text { Single Blind } \\
\text { Placebo controlled }\end{array}$ & DM-2 & $\begin{array}{c}3 \mathrm{gl} \text { of Black tea }+3 \mathrm{~g} \\
\text { cardamom vs. } 3 \text { gl black tea }+ \\
3 \mathrm{~g} \text { cinnamon } \\
3 \mathrm{gl} \text { black tea }+3 \mathrm{~g} \text { ginger vs. } \\
3 \text { gl black tea }+1 \mathrm{~g} \text { saffron vs. } \\
3 \text { gl black tea (control) } \\
\end{array}$ & 8 weeks & $\begin{array}{c}\text { FBG, t-chol, TG, } \\
\text { LDL-c, HDL-c, } \\
\text { HbA1c, }\end{array}$ & Yes & Yes \\
\hline $\begin{array}{c}\text { Azimi } 2016 \\
{[45]}\end{array}$ & 208 & $17 / 16 / 15 / 16 / 15$ & $54.33 \pm 0.5$ & $\begin{array}{c}\text { Parallel } \\
\text { Randomized } \\
\text { Single Blind } \\
\text { Placebo controlled }\end{array}$ & DM-2 & $\begin{array}{c}3 \mathrm{gl} \text { of black tea }+3 \mathrm{~g} \\
\text { cardamom vs. } 3 \text { gl black tea }+ \\
3 \mathrm{~g} \text { cinnamon vs. } 3 \text { gl black } \\
\text { tea }+3 \mathrm{~g} \text { ginger vs. } 3 \text { gl black } \\
\text { tea }+1 \mathrm{~g} \text { saffron vs. } 3 \text { gl black } \\
\text { tea (control) }\end{array}$ & 8 weeks & WC, SBP, DBP & Yes & Yes \\
\hline $\begin{array}{l}\text { Ebrahimi } \\
2019[46]\end{array}$ & 90 & 36 & $\begin{array}{l}55.2 \pm 7.3 \\
53 \pm 10.6\end{array}$ & $\begin{array}{c}\text { Prospective } \\
\text { DoubleBlind } \\
\text { Placebo Controlled } \\
\text { Randomized } \\
\end{array}$ & DM-2 & $\begin{array}{l}\text { Saffron } 100 \mathrm{mg} \text { vs. placebo } \\
100 \mathrm{mg} \text { maltodextrin }\end{array}$ & 12 weeks & SBP, DBP & Yes & Yes \\
\hline $\begin{array}{c}\text { Ebrahimi } \\
\text { 2019b [47] }\end{array}$ & 90 & 36 & $\begin{array}{l}55.2 \pm 7.3 \\
53 \pm 10.6\end{array}$ & $\begin{array}{c}\text { Prospective Double } \\
\text { Blind Placebo } \\
\text { Controlled } \\
\text { Randomized }\end{array}$ & DM-2 & $\begin{array}{l}\text { Saffron } 100 \mathrm{mg} \text { vs. placebo } \\
100 \mathrm{mg} \text { maltodextrin }\end{array}$ & 12 weeks & $\begin{array}{c}\text { FBG, HbA1c, } \\
\text { TG, t-chol, } \\
\text { HDL-c, } \\
\text { LDL-c, WC }\end{array}$ & Yes & Yes \\
\hline $\begin{array}{c}\text { Javandoost } \\
2017[52]\end{array}$ & 44 & 18 & $\begin{array}{c}44.50 \\
(24.75-51.50) \\
33.10 \\
(29.85-35.42) \\
\end{array}$ & $\begin{array}{l}\text { Double blind } \\
\text { randomized } \\
\text { placebo controlled }\end{array}$ & MS & $30 \mathrm{mg}$ crocin vs. placebo & 8 weeks & $\begin{array}{l}\text { FBG, TG, HDL-c, } \\
\text { LDL-c, } \\
\text { t-chol }\end{array}$ & No & No \\
\hline $\begin{array}{c}\text { Karimi } \\
\text { Nazari2019 } \\
{[56]}\end{array}$ & 80 & 27 & $\begin{array}{c}57.95 \pm 8.18 \\
57.9 \pm 8.7\end{array}$ & $\begin{array}{l}\text { Double blind } \\
\text { randomized } \\
\text { placebo controlled }\end{array}$ & Prediabetes & $15 \mathrm{mg}$ saffron vs. placebo & 8 weeks & $\begin{array}{l}\text { FBG, TG, HDL-c, } \\
\text { LDL-c, } \\
\text { t-chol }\end{array}$ & Yes & Yes \\
\hline $\begin{array}{l}\text { Kermani } \\
2017[53]\end{array}$ & 48 & 7 & $\begin{array}{l}53.8 \pm 9.2 \\
50.9 \pm 8.8\end{array}$ & $\begin{array}{l}\text { Double blind } \\
\text { Randomized } \\
\text { placebo controlled }\end{array}$ & MS & $100 \mathrm{mg}$ crocin vs. placebo & 6 weeks & $\begin{array}{l}\text { FBG, TG, HDL-c, } \\
\text { LDL-c, t-chol, } \\
\text { SBP, DBP, WC }\end{array}$ & No & No \\
\hline
\end{tabular}


Table 1. Cont.

\begin{tabular}{|c|c|c|c|c|c|c|c|c|c|c|}
\hline Study (Year) & Sample & $\begin{array}{l}\text { Gender } \\
\text { (Males) }\end{array}$ & Age (Years) & Study Design & Condition & Intervention/Groups & Duration & Outcome & $\begin{array}{l}\text { Assessment } \\
\text { of Diet }\end{array}$ & $\begin{array}{l}\text { Assessment } \\
\text { of Physical } \\
\text { Activity }\end{array}$ \\
\hline $\begin{array}{l}\text { Milajerdi } \\
2017[48]\end{array}$ & 54 & 12 & $\begin{array}{l}54.57 \pm 6.96 \\
55.42 \pm 7.58\end{array}$ & $\begin{array}{l}\text { Double blind } \\
\text { Randomized } \\
\text { placebo controlled }\end{array}$ & DM-2 & $30 \mathrm{mg}$ saffron vs. placebo & 8 weeks & $\begin{array}{l}\text { WC, SBP, DBP } \\
\text { (does not report } \\
\text { full data) }\end{array}$ & Yes & Yes \\
\hline $\begin{array}{l}\text { Milajerdi } \\
2018[49]\end{array}$ & 54 & 12 & $\begin{array}{l}54.57 \pm 6.96 \\
55.42 \pm 7.58\end{array}$ & $\begin{array}{c}\text { Triple blind } \\
\text { randomized } \\
\text { placebo controlled }\end{array}$ & DM-2 & $30 \mathrm{mg}$ saffron vs. placebo & 8 weeks & $\begin{array}{c}\text { FBG, t-chol, TG, } \\
\text { HDL-c, LDL-c, } \\
\text { HbA1c }\end{array}$ & Yes & Yes \\
\hline $\begin{array}{c}\text { Moravej } \\
\text { Aleali 2019 } \\
{[50]}\end{array}$ & 64 & 19 & $\begin{array}{l}52.4 \pm 13 \\
53.5 \pm 9.9\end{array}$ & $\begin{array}{c}\text { Double blind } \\
\text { Randomized } \\
\text { placebo controlled }\end{array}$ & DM-2 & $30 \mathrm{mg}$ saffron vs. placebo & 3 months & $\begin{array}{l}\text { FBG, t-chol, TG, } \\
\text { HDL-c, LDL-c, } \\
\text { HbA1c, }\end{array}$ & Yes & No \\
\hline $\begin{array}{l}\text { Nikbakht-Jam } \\
2016[54]\end{array}$ & 60 & 25 & $\begin{array}{l}38.97 \pm 13.33 \\
43.46 \pm 12.77\end{array}$ & $\begin{array}{l}\text { Double blind } \\
\text { Randomized } \\
\text { placebo controlled }\end{array}$ & $\begin{array}{c}\text { MS (DM } \\
16 \%)\end{array}$ & $30 \mathrm{mg}$ crocin vs. placebo & 8 weeks & $\begin{array}{l}\text { FBG, t-chol, TG, } \\
\text { HDL-c, LDL }\end{array}$ & No & No \\
\hline $\begin{array}{l}\text { Zilaee } 2018 \\
\quad[55]\end{array}$ & 76 & 9 & $\begin{array}{l}42.19 \pm 11.52 \\
43.60 \pm 9.05\end{array}$ & $\begin{array}{c}\text { Double blind } \\
\text { Randomized } \\
\text { placebo controlled }\end{array}$ & MS & $100 \mathrm{mg}$ saffron vs. placebo & 12 weeks & $\begin{array}{l}\text { LDL-c, } \\
\text { HDL-c, TG, } \\
\text { t-chol, WC }\end{array}$ & No & $\begin{array}{c}\text { Yes } \\
\text { (self-reported) }\end{array}$ \\
\hline $\begin{array}{l}\text { Sepahi } 2018 \\
{[51]}\end{array}$ & 60 & 29 & $\begin{array}{l}53.31 \pm 6.6 \\
56.09 \pm 4.3 \\
57.17 \pm 2.9\end{array}$ & $\begin{array}{c}\text { Double masked } \\
\text { Randomized phase } \\
2 \text { placebo controlled }\end{array}$ & $\begin{array}{l}\text { DM-1:10 } \\
\text { DM-2:50 }\end{array}$ & $\begin{array}{c}\text { Crocin } 5 \text { mg vs. crocin } 15 \mathrm{mg} \\
\text { vs. placebo }\end{array}$ & 3 months & $\begin{array}{l}\text { FBG, HbA1c } \\
\text { HDL-c, } \\
\text { LDL-c, TG, } \\
\text { t-Chol }\end{array}$ & No & No \\
\hline
\end{tabular}

Abbreviations used: DM-2 (diabetes mellitus type 2), FBG (fasting blood glucose), t-chol (total cholesterol), HDL-c (high-density lipoproteincholesterol), LDL-c (low-density lipoproteincholesterol), MS (metabolic syndrome), TG (triglycerides), WC (waist circumference), HbA1c (glycated haemoglobin), SBP (systolic blood pressure), DBP (diastolic blood pressure), gl (glasses). 


\subsection{Risk of Bias Assessment}

All studies reported the randomization technique that was followed. One study [53] reported allocation methods that imply blinding of personnel could have been broken, so this was considered as of high risk of bias in allocation concealment. Studies that provided insufficient information about allocation methods were considered asexhibiting unclear risk of bias [44,45,54,55]. Two single- blinded studies [44,45] were reported as being of high risk, and other studies [53-55] were characterized as demonstrating unclear risk as their blinding method was not mentioned, although reported as double-blinded. In relation to blinding of outcome assessment, five studies [53-55,57] were alsocharacterized as having an unclear risk because they were mentioned as double-blind but did not provide sufficient details on the method and two [44,45] were judged as exhibiting a high risk because they were single-blinded. All others were assessed as low-risk studies providing adequate evidence. Two studies [54,57] were considered of unclear risk because they did not address what missing data they had, and one [53] was of high risk due to high percentage of dropout (20\%) that may have influenced their results. Except for one [55], all studies prespecified their primary and secondary endpoints with trial registration, so their judgement for selective reporting was considered low risk. Other biases were considered, such as not reporting assessment of diet and physical activity, if the company that produced the product was funding the trial or if there were any methodological issues that were not included in the mentioned bias. Figure 2 shows the risk of bias assessment across all included studies and Figure 3 shows the risk of bias summary, including assessment of each risk item of all included studies.

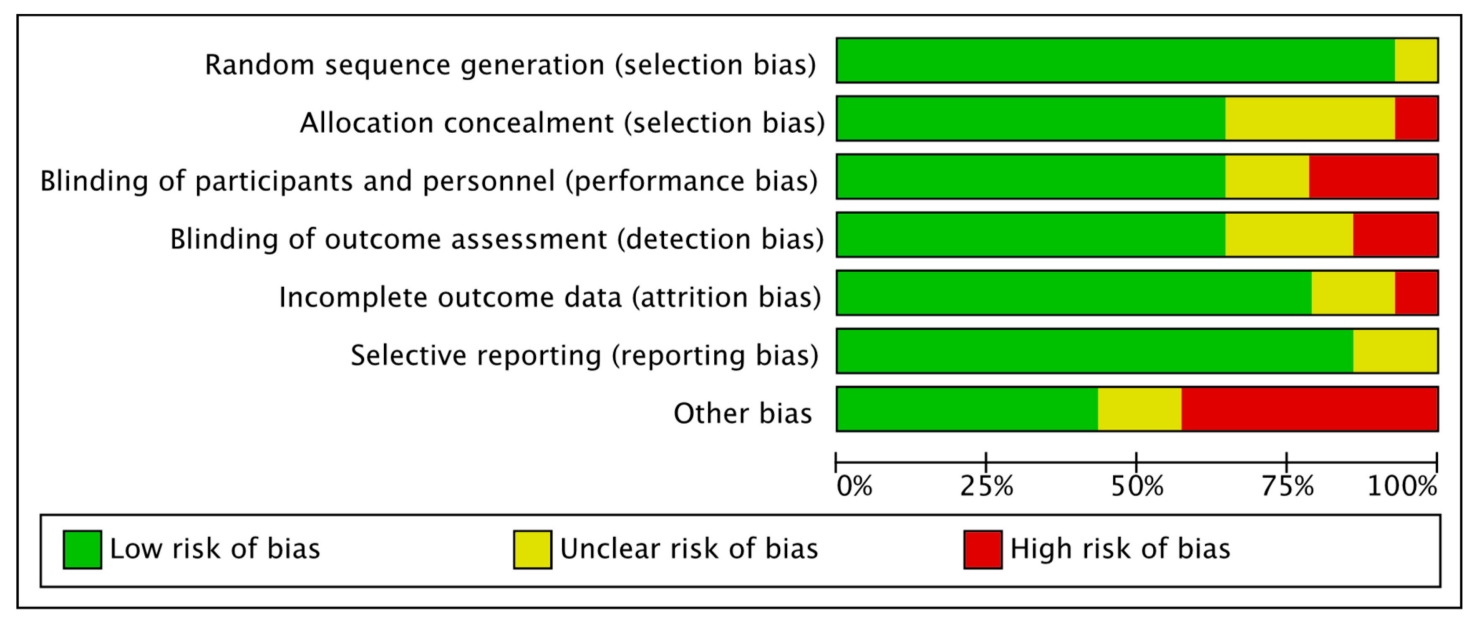

Figure 2. Risk of Bias assessment summary. 


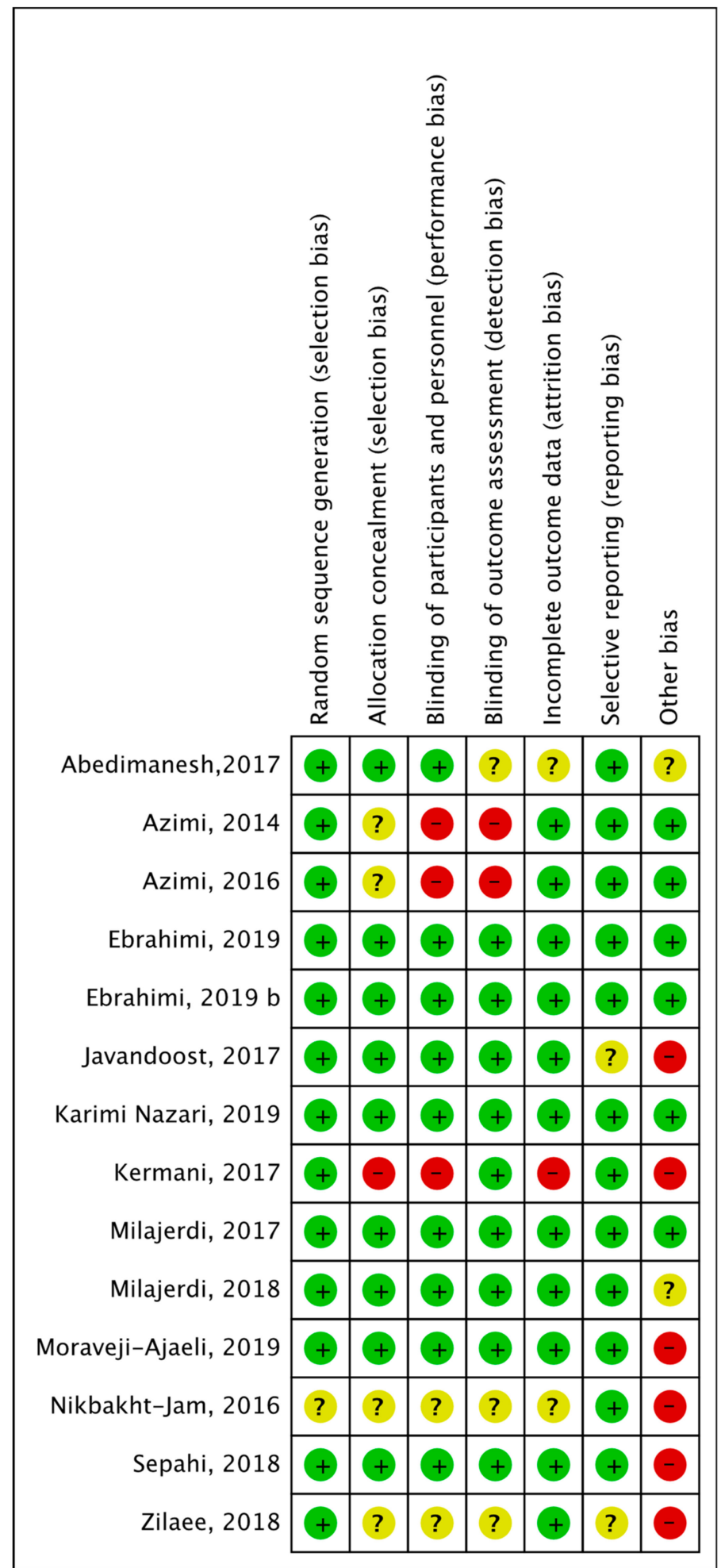

Figure 3. Risk of bias assessment summary for each included study.

\subsection{Glycemic Control}

Only three studies $[49,50,56]$ out of 10that examined FBG as a primary outcome showed a significant reduction in FBG ( $p<0.001 ; p=0.013 ; p=0.005$ respectively) after oral administration of saffron or crocin versus placebo (Table 2). Out of those three studies, only Karimi et al. demonstrated also a significant reduction $(p<0.005)$ in HbA1c. Moreover, Sepahi et al. showed a significant reduction 
( $p=0.024)$ of $\mathrm{HbA} 1 \mathrm{c}$ only in one of the two intervention arms (15 mg crocin) compared to the placebo arm (Table 3).

Table 2. Summary of findings for fasting blood glucose (FBG).

\begin{tabular}{|c|c|c|c|c|c|c|c|c|c|c|c|}
\hline \multirow{3}{*}{ Study Name, Year } & \multicolumn{6}{|c|}{ Intervention Group } & \multicolumn{4}{|c|}{ Control Group } & \multirow[b]{3}{*}{$p$} \\
\hline & \multicolumn{3}{|c|}{ Baseline } & \multicolumn{3}{|c|}{ Post-Intervention } & \multicolumn{2}{|c|}{ Baseline } & \multicolumn{2}{|c|}{ Post-Interventior } & \\
\hline & $N$ & Mean & SD & Mean & SD & $N$ & Mean & SD & Mean & SD & \\
\hline Abedimanesh 2017 \# [57] & 25 & 107.25 & 44.14 & 100.85 & 26.49 & 25 & 123.11 & 59.31 & 117.63 & 56.48 & NS \\
\hline Azimi 2014 \# [44] & 42 & 358.35 & 4.3 & 356.66 & 4.39 & 39 & 355.28 & 11.86 & 353.23 & 11.96 & NS \\
\hline Ebrahimi 2019b [47] & 40 & 166.7 & 53.7 & 162.1 & 52.7 & 40 & 160.9 & 51.9 & 147.7 & 51.8 & NS \\
\hline Kermani 2017 \# [53] & 24 & 110 & 42.16 & 111.2 & 35.6 & 24 & 124.4 & 47.7 & 129.3 & 75 & NS \\
\hline Milajerdi 2018 [49] & 26 & 164.36 & 40.88 & 128.84 & 31.86 & 26 & 159.64 & 38.38 & 153.76 & 41.23 & $<0.001$ \\
\hline Moravej Aleali 2019 [50] & 32 & 173.2 & 73.9 & 147.9 & 53.5 & 32 & 177.1 & 60.1 & 188.5 & 74.7 & 0.013 \\
\hline Nikbakht-Jam 2016 \# [54] & 30 & 102.34 & 36.88 & 104.52 & 49.2 & 30 & 101.31 & 29.08 & 103.31 & 25.18 & NS \\
\hline Sepahi 2018 [51] & 55 & 176.6 & 64.17 & 155.39 & 55.49 & 23 & 175.15 & 7.38 & 169.45 & 7.61 & NS \\
\hline
\end{tabular}

Abbreviations used: SD, standard deviation; NS (not significant), $p<0.05$ was considered statistically significant. \# study of low quality according to risk of bias assessment.

Table 3. Summary of findings for glycated hemoglobin(HbA1c).

\begin{tabular}{|c|c|c|c|c|c|c|c|c|c|c|c|}
\hline \multirow{3}{*}{ Study Name, Year } & \multicolumn{6}{|c|}{ Intervention Group } & \multicolumn{4}{|c|}{ Control Group } & \multirow[b]{3}{*}{$p$} \\
\hline & \multicolumn{3}{|c|}{ Baseline } & \multicolumn{3}{|c|}{ Post-Intervention } & \multicolumn{2}{|c|}{ Baseline } & \multicolumn{2}{|c|}{ Post-Interventior } & \\
\hline & $N$ & Mean & SD & Mean & SD & $N$ & Mean & SD & Mean & SD & \\
\hline Azimi 2014 \# [44] & 42 & 7.73 & 0.07 & 7.74 & 0.07 & 39 & 7.5 & 0.1 & 7.51 & 0.1 & NS \\
\hline Ebrahimi 2019b [47] & 40 & 8.01 & 1.4 & 7.69 & 1.49 & 40 & 7.38 & 1.53 & 7.34 & 1.48 & NS \\
\hline Karimi Nazari 2019 [56] & 36 & 5.85 & 0.12 & 5.7 & 0.11 & 39 & 5.88 & 0.11 & 5.92 & 0.12 & $<0.005$ \\
\hline Milajerdi 2018 [49] & 26 & 6.37 & 1.3 & 6.75 & 1.28 & 26 & 6.83 & 1.36 & 7.25 & 1.65 & NS \\
\hline Moravej Aleali 2019 [50] & 32 & 8.9 & 2 & 8.2 & 1.8 & 32 & 8.8 & 1.8 & 8.3 & 1.4 & NS \\
\hline Sepahi 2018 [51] & 23 & 8.17 & 0.11 & 7.29 & 0.12 & 23 & 8.15 & 0.22 & 8.03 & 0.14 & 0.024 * \\
\hline
\end{tabular}

\subsection{Lipid Profile}

Total cholesterol concentration was significantly reduced after the intervention in only three studies $[44,50,53]$ out of 11 . It is notworthy, that Kermani et al. [53] found a significant reduction in $\mathrm{t}$-cholconcentration both in the intervention $(p<0.001)$ and the placebo groups $(p=0.01)$ at the end of the study, but there was not a significant difference in the magnitude of reduction between the two groups (Table 4). On the other hand, Azimi et al. [44] and Moravej-Aleali et al. [50] found significant differences in the reduction of $\mathrm{t}$-cholconcentration $(p=0.004 ; p=0.014$ respectively) and LDL-concentration ( $p=0.01 ; p=0.014$ respectively) between their two groups. Zilaee et al. [55] showed a significant reduction in LDL-concentration after the intervention $(p=0.03)$ between the two groups (saffron group vs. placebo group), whilst Nikbakth [54] showed a reduction only between baseline and post intervention in the crocin arm $(p=0.02)$, whereas the magnitude of reduction was not significant between the two arms (Table 5). Azimi et al. [44], found a significant difference in HDL-c $(p=0.001)$ as well. In a study by Javandoost et al. [52], HDL-c increased significantly after the intervention both in the crocin and the placebo arm ( $p=0.004 ; p<0.001$ respectively) (Table 6). A significant post-intervention reduction $(p<0.003)$ was seen in TG only byKermani et al. [52], and only in the crocin group (Table 7). 
Table 4. Summary of findings for total cholesterol ( $\mathrm{t}-\mathrm{chol})$.

\begin{tabular}{|c|c|c|c|c|c|c|c|c|c|c|c|}
\hline \multirow{3}{*}{ Study Name, Year } & \multicolumn{6}{|c|}{ Intervention Group } & \multicolumn{4}{|c|}{ Control Group } & \multirow[b]{3}{*}{$p$} \\
\hline & \multicolumn{3}{|c|}{ Baseline } & \multicolumn{2}{|c|}{ Post-Intervention } & & \multicolumn{2}{|c|}{ Baseline } & \multicolumn{2}{|c|}{ Post-Intervention } & \\
\hline & $N$ & Mean & SD & Mean & SD & $N$ & Mean & SD & Mean & SD & \\
\hline Ebrahimi 2019b [47] & 40 & 143.7 & 36.6 & 152.8 & 31.4 & 40 & 147 & 32.5 & 155.1 & 37.2 & NS \\
\hline Karimi Nazari 2019 [56] & 36 & 186.67 & 17.22 & 184.54 & 17.45 & 39 & 192.69 & 13.57 & 190.88 & 14.6 & NS \\
\hline Javandoost 2017 [52] & 22 & 232.18 & 66.52 & 220.09 & 55.6 & 22 & 209.19 & 38.41 & 199.95 & 50.1 & NS \\
\hline Moravej Aleali 2019 [50] & 32 & 169.3 & 38.8 & 152.9 & 32.1 & 32 & 152.21 & 31.5 & 164.2 & 43.5 & 0.014 \\
\hline Nikbakht-Jam 2016 \# [54] & 30 & 224.48 & 60.83 & 210.52 & 52.68 & 30 & 212.76 & 37.82 & 210.9 & 50.3 & NS \\
\hline Sepahi 2018 [51] & 55 & 196.54 & 55.2 & 199.02 & 49.5 & 23 & 189.45 & 7.24 & 190.85 & 7.17 & NS \\
\hline Zilaee 2018 \# [55] & 30 & 199.15 & 27.3 & 96.88 & 37.73 & 31 & 177.16 & 33.34 & 167.36 & 37.03 & NS \\
\hline
\end{tabular}

Abbreviations used: NS (not significant), $p<0.05$ was considered statistically significant, ${ }^{\mathrm{a}}(p<0.001),{ }^{\mathrm{b}}(p=0.01)$.

\# study of low quality according to risk of bias assessment.

Table 5. Summary of findings for LDL cholesterol (LDL-c).

\begin{tabular}{|c|c|c|c|c|c|c|c|c|c|c|c|}
\hline \multirow{3}{*}{ Study Name, Year } & \multicolumn{5}{|c|}{ Intervention Group } & \multicolumn{6}{|c|}{ Control Group } \\
\hline & \multirow[b]{2}{*}{$N$} & \multicolumn{2}{|c|}{ Baseline } & \multicolumn{2}{|c|}{ Post-Intervention } & \multirow[b]{2}{*}{$N$} & \multicolumn{2}{|c|}{ Baseline } & \multicolumn{2}{|c|}{ Post-Intervention } & \multirow[b]{2}{*}{$p$} \\
\hline & & Mean & SD & Mean & SD & & Mean & SD & Mean & SD & \\
\hline Abedimanesh 2017 \# [57] & 25 & 94.1 & 35.4 & 89.2 & 32.34 & 25 & 81.31 & 28.47 & 83.21 & 26.23 & NS \\
\hline Azimi 2014 \# [44] & 42 & 229.57 & 2.64 & 228.28 & 2.63 & 39 & 208.64 & 6.06 & 205.94 & 5.51 & 0.01 \\
\hline Ebrahimi 2019b [47] & 40 & 82.7 & 25.7 & 89.5 & 23.9 & 40 & 84.5 & 26.6 & 90.7 & 30.5 & NS \\
\hline Karimi Nazari 2019 [56] & 36 & 114.75 & 13.25 & 113.55 & 12.77 & 39 & 120.31 & 12.69 & 117.72 & 11.34 & NS \\
\hline Javandoost 2017 [52] & 21 & 162.67 & 66.78 & 131.25 & 54.66 & 22 & 130.92 & 39.8 & 116.17 & 70.5 & NS \\
\hline Kermani 2017 \# [53] & 24 & 146.3 & 25.4 & 139.1 & 25.8 & 24 & 147.2 & 44.9 & 127.5 & 32.1 & NS \\
\hline Milajerdi 2018 [49] & 26 & 83.79 & 29.48 & 85.9 & 32.04 & 26 & 95.9 & 36.16 & 82.94 & 26.95 & NS \\
\hline Moravej Aleali 2019 [50] & 32 & 87.7 & 26.1 & 72.9 & 26.2 & 32 & 81.2 & 25.4 & 82.9 & 40.5 & 0.014 \\
\hline Nikbakht-Jam 2016 \# [54] & 30 & $152.29^{a}$ & 56.93 & $123.52^{\mathrm{a}}$ & 48.06 & 30 & 138.45 & 36.76 & 125.76 & 52.16 & NS \\
\hline Sepahi 2018 [51] & 55 & 120.99 & 43.15 & 118.54 & 46.13 & 23 & 113.85 & 6.02 & 110.45 & 5.31 & NS \\
\hline Zilaee 2018 \# [55] & 30 & 120.03 & 30.01 & 97.65 & 25.88 & 31 & 125.16 & 22.33 & 113 & 26.56 & 0.03 \\
\hline
\end{tabular}

Abbreviations used: NS (not significant), $p<0.05$ was considered statistically significant, ${ }^{a}(p=0.02)$. \# study of low quality according to risk of bias assessment.

Table 6. Summary of findings for HDL cholesterol (HDL-c).

\begin{tabular}{|c|c|c|c|c|c|c|c|c|c|c|c|}
\hline \multirow{3}{*}{ Study Name, Year } & \multicolumn{5}{|c|}{ Intervention Group } & \multicolumn{6}{|c|}{ Control Group } \\
\hline & \multirow[b]{2}{*}{$N$} & \multicolumn{2}{|c|}{ Baseline } & \multicolumn{2}{|c|}{ Post-Intervention } & \multirow[b]{2}{*}{$N$} & \multicolumn{2}{|c|}{ Baseline } & \multicolumn{2}{|c|}{ Post-Intervention } & \multirow[b]{2}{*}{$p$} \\
\hline & & Mean & SD & Mean & SD & & Mean & SD & Mean & SD & \\
\hline Azimi2014 \# [44] & 42 & 53.97 & 0.71 & 54.76 & 0.74 & 39 & 50.38 & 1.38 & 51.53 & 1.52 & 0.001 \\
\hline Ebrahimi 2019b [47] & 40 & 41.8 & 8.4 & 42.2 & 9.4 & 40 & 44.35 & 10.1 & 44.9 & 11.3 & NS \\
\hline Karimi Nazari 2019 [56] & 36 & 49.97 & 11.62 & 50.25 & 11.15 & 39 & 52.2 & 8.8 & 52 & 9.8 & NS \\
\hline Milajerdi 2018 [49] & 26 & 58.83 & 8.47 & 63.33 & 5.11 & 26 & 60.95 & 7.17 & 6.17 & 7.08 & NS \\
\hline Moravej Aleali 2019 [50] & 32 & 45.1 & 9.1 & 48.2 & 10.6 & 32 & 38.3 & 9.6 & 43 & 11.1 & NS \\
\hline Nikbakht-Jam 2016 \# [54] & 30 & 38.59 & 10.14 & 49.25 & 11.5 & 30 & 38.93 & 9.18 & 51.24 & 10.44 & NS \\
\hline Sepahi 2018 [51] & 55 & 43.57 & 11.53 & 42.94 & 10.3 & 23 & 43.95 & 0.94 & 44.35 & 0.85 & NS \\
\hline Zilaee 2018 \# [55] & 30 & 39.03 & 5.34 & 43 & 9.97 & 31 & 39.13 & 8 & 43.46 & 6.5 & NS \\
\hline
\end{tabular}

Abbreviations used: NS (not significant), $p<0.05$ was considered statistically significant, ${ }^{a}(p=0.004),{ }^{\mathrm{b}}(p<0.001)$.

\# study of low quality according to risk of bias assessment. 
Table 7. Summary of findings for triglycerides (TG).

\begin{tabular}{|c|c|c|c|c|c|c|c|c|c|c|c|}
\hline \multirow{3}{*}{ Study Name, Year } & \multicolumn{6}{|c|}{ Intervention Group } & \multicolumn{5}{|c|}{ Control Group } \\
\hline & \multirow[b]{2}{*}{$N$} & \multicolumn{2}{|c|}{ Baseline } & \multicolumn{3}{|c|}{ Post-Intervention } & \multicolumn{2}{|c|}{ Baseline } & \multicolumn{3}{|c|}{ Post-Intervention } \\
\hline & & Mean & SD & Mean & SD & $N$ & Mean & SD & Mean & SD & $p$ \\
\hline Abedimanesh 2017 \# [57] & 25 & 200.05 & 74.08 & 193.05 & 60.44 & 25 & 182.37 & 87.27 & 192.32 & 101 & NS \\
\hline Azimi 2014 \# [44] & 42 & 391.88 & 3.91 & 390.71 & 3.82 & 39 & 386.54 & 13.28 & 382.48 & 12.72 & NS \\
\hline Ebrahimi 2019b [47] & 40 & 165.8 & 121.8 & 175 & 98.1 & 40 & 170.4 & 63.5 & 168 & 58.3 & NS \\
\hline Karimi Nazari 2019 [56] & 36 & 101.5 & 20.34 & 100.22 & 17.63 & 39 & 108.94 & 18.2 & 107.84 & 15.97 & NS \\
\hline Javandoost 2017 [52] & 21 & 155.58 & 73.74 & 163.25 & 99.58 & 22 & 164.33 & 84.8 & 153.67 & 75.29 & NS \\
\hline Kermani 2017 \# [53] & 24 & $218.1^{\mathrm{a}}$ & 80 & $173.8^{\mathrm{a}}$ & 97.5 & 24 & 232.4 & 83.2 & 197.5 & 82.9 & NS \\
\hline Milajerdi 2018 [49] & 26 & 146.54 & 41.86 & 127 & 37.61 & 26 & 137.96 & 40.71 & 128.2 & 38.5 & NS \\
\hline Moravej Aleali 2019 [50] & 32 & 166.4 & 87.7 & 156.4 & 73.2 & 32 & 187.2 & 137.1 & 191.8 & 135.3 & NS \\
\hline Nikbakht-Jam 2016 \# [54] & 30 & 153.17 & 67.06 & 147 & 72.52 & 30 & 165.47 & 76.73 & 153.9 & 89.9 & NS \\
\hline Sepahi 2018 [51] & 55 & 199.65 & 112.24 & 196.32 & 97.6 & 23 & 203.75 & 9.32 & 200.7 & 8.09 & NS \\
\hline Zilaee2018 \# [55] & 30 & 139,76 & 70,14 & 96,88 & 37,73 & 31 & 139 & 73,52 & 107,6 & 43,98 & NS \\
\hline
\end{tabular}

Abbreviations used: NS (not significant), $p<0.05$ was considered statistically significant, ${ }^{\text {a }}(p<0.003)$. \# study of

low quality according to Risk of Bias Assessment.

\subsection{Other Components of Metabolic Syndrome (MS)}

Ebrahimi et al., [46] found a significant difference in the reduction $(p=0.005)$ of SBP between the two groups (Table 8) but not of DBP (Table 9).Furthermore, a significant reduction in WC was observed only in the study of Ebrahimi et al. [47] $(p<0.001)$ in the saffron group versus placebo (Table 10).

Table 8. Summary of findings for systolic blood pressure (SBP).

\begin{tabular}{|c|c|c|c|c|c|c|c|c|c|c|c|}
\hline \multirow{3}{*}{ Study Name, Year } & \multicolumn{6}{|c|}{ Intervention Group } & \multicolumn{4}{|c|}{ Control Group } & \multirow[b]{3}{*}{$p$} \\
\hline & \multicolumn{3}{|c|}{ Baseline } & \multicolumn{2}{|c|}{ Post-Intervention } & & \multicolumn{2}{|c|}{ Baseline } & \multicolumn{2}{|c|}{ Post-Intervention } & \\
\hline & $N$ & Mean & SD & Mean & SD & $N$ & Mean & SD & Mean & SD & \\
\hline Ebrahimi 2019 [46] & 40 & 132.7 & 21.3 & 124.5 & 13.2 & 40 & 127.4 & 15.3 & 128.3 & 12.4 & 0.005 \\
\hline Kermani 2017 \# [53] & 24 & 129.3 & 16.9 & 126.8 & 19.4 & 24 & 131 & 14 & 131.8 & 13.5 & NS \\
\hline
\end{tabular}

Abbreviations used: NS (not significant), $p<0.05$ was considered statistically significant. \# study of low quality according to risk of bias assessment.

Table 9. Summary of findings for diastolic blood pressure (DBP).

\begin{tabular}{|c|c|c|c|c|c|c|c|c|c|c|c|}
\hline \multirow{2}{*}{ Study Name, Year } & \multicolumn{5}{|c|}{ Intervention Group } & \multicolumn{6}{|c|}{ Control Group } \\
\hline & $N$ & Mean & SD & Mean & SD & $N$ & Mean & SD & Mean & SD & $p$ \\
\hline Ebrahimi 2019 [46] & 40 & 79.5 & 10.8 & 76.7 & 9.9 & 40 & 79.7 & 11.1 & 75.9 & 14 & NS \\
\hline Kermani 2017 \# [53] & 24 & 81.1 & 12.8 & 80.9 & 14.5 & 24 & 85 & 20.6 & 84.1 & 13.4 & NS \\
\hline
\end{tabular}

Abbreviations used: NS (not significant), $p<0.05$ was considered statistically significant. \# study of low quality according to risk of bias assessment.

Table 10. Summary of findings for waist circumference (WC).

\begin{tabular}{|c|c|c|c|c|c|c|c|c|c|c|c|}
\hline \multirow{2}{*}{ Study Name, Year } & \multicolumn{5}{|c|}{ Intervention Group } & \multicolumn{6}{|c|}{ Control Group } \\
\hline & $N$ & Mean & SD & Mean & SD & $N$ & Mean & SD & Mean & SD & $p$ \\
\hline Abedimanesh 2017 \# [57] & 25 & 95 & 12.85 & 92.68 & 13.03 & 25 & 92.84 & 9.13 & 91.42 & 8.94 & NS \\
\hline Azimi 2016 \# [45] & 42 & 102.26 & 1.5 & 102.02 & 1.5 & 39 & 100.92 & 1.4 & 100.66 & 1.4 & NS \\
\hline Ebrahimi 2019b [47] & 40 & 104.31 & 7.85 & 100.02 & 7.32 & 40 & 102.95 & 7.94 & 104.33 & 7.42 & $<0.001$ \\
\hline
\end{tabular}

Abbreviations used: NS (not significant), $p<0.05$ was considered statistically significant. \# study of low quality according to risk of bias assessment. 


\section{Discussion}

This systematic review includes 14RCTs that investigated the impact of saffron administration and its bioactive ingredient crocin on the metabolic profile in patients with DM and MS. In particular, the following clinical outcomes: FBG, HbA1c, t-chol, LDL-c, HDL-c, TG, WC, SBP, and DBP were documented.

Some of the studies we examined provided significant differences in the lipid profile either between the intervention and placebo group or within the groups [44,53-55]. These results were very inconclusive and the studies they came from had a non-favorable risk of bias, so they were excluded from our conclusions.

According to the risk of bias assessment, eight studies [46-52,56] were not of low quality (i.e., based on Cochrane criteria for considering a study of low quality: it should have a judgement on high risk of bias $\geq 2$ items or unclear risk of bias $\geq 3$ items). One of them [52] did not assess dietary intake and physical activity, whilst two of them [50,51] did not assess physical activity during the intervention. This may result in non-reliable conclusions because possible changes in physical activity or/and dietary intake throughout the period of intervention may influence the above metabolic parameters. In our view, conclusions of this systematic review should include and interpret only results from high-quality studies that assessed dietary intake and physical activity and did not demonstrate any significant differences in these parameters between baseline and post-intervention. Only five studies $[46-49,56]$ in our search met these standards.

Ebrahimi and his colleagues [46] only found a significant difference in DBP and not in SBP after supplementation of $100 \mathrm{mg}$ saffron versus placebo in DM-2 patients for 12weeks. In another paper, the same investigators [47] did not find any significant difference in lipid profile and glycemic control but found significant difference in weight and WC. This may be explained by the findings from Gout et al. [58], who administrated $176.5 \mathrm{mg}$ of saffron extract to mildly overweight healthy women and found a reduction of snacking and longer lasting satiety which might have contributed to weight loss. Karimi et al., found a significant difference in FBG and HbA1c in prediabetic overweight/obese patients after supplementation with $15 \mathrm{mg}$ saffron for eight weeks, but no overall effect on the lipid profile. Furthermore, Milajerdi et al. [48,49], found no significant differences in blood pressure, lipid profile and $\mathrm{HbA1c}$, but FBG was significantly decreased in the intervention arm after administration of $30 \mathrm{mg}$ saffron extract in DM-2 patients for eight weeks. This agrees with the findings of a meta-analysis from Pourmasoumi et al. [59] that reports no clinical benefit on the lipid profile, but a beneficial effect of saffron on FBG and $\mathrm{HbA1c}$. Our qualitative findings are in accordance with their conclusions, although in their analysis they did not focus on DM and MS and also included healthy subjects and populations with other diseases. Furthermore, they did not include six studies $[46,47,50,52,55,56]$ that we considered in this review.

The findings presented in this systematic review are, in part, not in line with previous research in this area with animal models. There is abundant evidence in vitro and in vivo animal studies that supplementation of saffron and its bioactive ingredients have a beneficial effect on the lipid profile and blood pressure [17-26]. Potential mechanisms of the effect on the lipid profile may be a potential inhibitory action of saffron and its bioactive ingredients on pancreatic lipase [60], antioxidant action, increase of the levels of adiponectin, activation of peroxisome proliferator-activated receptor alpha (PPAR $\alpha$ )and modulation of heat shock proteins. Hypotensive effects can be attributed to blocking of calcium channels and possible interaction with endothelial nitric oxide (NO) [61]. Our findings do not match the results from animal studies, possibly due to varying doses and small duration of administration in humans. Nevertheless, regarding FBG, evidence from animal models [8-13] is in accordance with our findings on humans $[47,56]$.

It is plausible that a number of limitations might have influenced the results of this systematic review. As mentioned above, there is a limited number of high-quality studies examining the effects of saffron on humans without major methodological issues. There is high heterogeneity in the studiesincluded, mainly regarding the amount and form of supplement 
used. As Tomé-Carneiro et al. [62] mentioned, a significant parameter in nutraceutical research is bioavailability. Different preparation methods do not supply an obtainable form of nutraceuticals. There is no clear evidence on the bioavailability and absorption in the gastrointestinal tract of saffron and its bioactive constituents. From what is shown in recent bibliography, antioxidant supplements do not work exactly how we were hoping. As has been shown in a systematic review and meta-analysis of 68 RCTs [63] examining the effect of antioxidant supplements on mortality, primary and secondary prevention of diseases, supplements are not effective in reducing oxidative stress and mortality. Although in vitro trials show the effectiveness of antioxidants, this does not apply in vivo as it has been shown that antioxidant molecules are not effective, due to kinetic constraints and limited bioavailability [64]. Another limitation of the current literature review is that there is no report of titration in the administered supplements. Additionally, there is limited evidence of the impact of saffron and its constituents on the metabolic profile in patients with DM and MS because the existing studies come from a limited number of research groups with few different study protocols, despite the number of published papers.

Moreover, the study populations are only Caucasians, an important limitation, because the etiology of DM2 differs among ethnic groups. Furthermore, another limitation is that we included study populations with DM and MS which were analyzed as one group in this review, not considering $\beta$ - cell dysfunction as a pathophysiological mechanism of DM, like insulin resistance [65].

Our study emphasizes on patients with impaired glucose tolerance and DM, but in the existing literature we found scarce evidence and there is high heterogeneity in the results of clinical trials. Therefore, we did not proceed to quantitative analysis and meta-analysis like Pourmasoumi but assessed more qualitatively existing trials.

To the best of our knowledge, this is the first systematic review investigating the impact of saffron on metabolic parameters in patients with DM and MS. One strength of our review is to focus only on patients with DM and MS. Another strength is that we included in the interpretation of our results the information whether dietary and physical activity (being two important cofounders) assessments were performed during the intervention period.

We propose that there is a need to conduct more high-quality clinical trials with different ethnic groups in order to investigate the potential beneficial impact of saffron supplementation on glycemic control and lipid profile of DM and MS patients in order to establish whether saffron could be a possible adjunct to diabetes therapy.

\section{Conclusions}

In summary, findings from this review are implausible due to the low-quality clinical trials assessed. It may be a favorable effect of saffron in FBG, but further research needs to be carried out in populations with greater homogeneity, different ethnic groups, more particular doses, and duration of supplementation. Also, it is necessary for the titration of the supplement used to provide more consistent results.

Supplementary Materials: The following are available online at http://www.mdpi.com/2072-6643/12/5/1424/s1, PPRISMA 2009 Checklist.

Author Contributions: Conceptualization, P.G., T.D. Methodology, P.G., E.K., Writing-Original Draft Preparation P.G., E.K., Writing-Review and Editing P.G., E.K., M.C., A.H., T.D. All authors have read and agreed to the published version of the manuscript.

Funding: This research received no external funding.

Conflicts of Interest: There are no conflicts of interest related to this publication. 


\section{Abbreviations}

$\begin{array}{ll}\text { AGEs } & \text { advanced glycation end product } \\ \text { PPARa } & \text { peroxisome proliferator-activated receptor alpha } \\ \text { BP } & \text { blood pressure } \\ \text { CK } & \text { creatine kinase } \\ \text { DM } & \text { Diabetes mellitus } \\ \text { FBG } & \text { fasting blood glucose } \\ \text { HbA1c } & \text { Glycatedhaemoglobin } \\ \text { HDL-C } & \text { high-density cholesterol } \\ \text { LDH } & \text { levels of lactate dehydrogenase } \\ \text { LDL-C } & \text { low-density cholesterol } \\ \text { MDA } & \text { malondialdehyde } \\ \text { MS } & \text { metabolic syndrome } \\ \text { NO } & \text { nitric oxide } \\ \text { TG } & \text { triglycerides } \\ \text { SOD } & \text { superoxide dismutase } \\ \text { t-chol } & \text { total serum cholesterol } \\ \text { WC } & \text { waist circumference } \\ \end{array}$

\section{References}

1. Didangelos, T.; Karamitsos, D. Chronic diabetic complications. In Diabetologia, Theory and Practice in the Treatment of Diabetes Mellitus, 2nd ed.; Karamitsos, D., Ed.; Siokis, Medical and Scientific Publications: Athens, Greece, 2009; Volume 1, pp. 429-535.

2. Calcutt, N.A.; Cooper, M.E.; Kern, T.S.; Schmidt, A.M. Therapies for hyperglycaemia-induced diabetic complications: From animal models to clinical trials. Nat. Rev. Drug Discov. 2009, 8, 417-430. [CrossRef]

3. Trisha, D. Preiodontal disease, the overlooked the diabetes complication. Nephrol. Nurs. J. 2009, 36, 489-495.

4. Brownlee, M. A radical explanation for glucose-induced $\beta$ cell dysfunction. J. Clin. Investig. 2003, 112, 1788-1790. [CrossRef]

5. Seino, Y.; Ueno, S.; Yabe, D.; Suzuki, A. Dietary recommendations for type 2 diabetes patients: Lessons from recent clinical and basic research in Asia. J. Diabetes Investig. 2019, 10, 1405-1407. [CrossRef] [PubMed]

6. Melnyk, J.P.; Wang, S.; Marcone, M.F. Chemical and biological properties of the world's most expensive spice: Saffron. Food Res. Int. 2010, 43, 1981-1989. [CrossRef]

7. Christodoulou, E.; Kadoglou, N.P.; Kostomitsopoulos, N.; Valsami, G. Saffron: A natural product with potential pharmaceutical applications. J. Pharm. Pharmacol. 2015, 67, 1634-1649. [CrossRef] [PubMed]

8. Schmidt, M.; Betti, G.; Hensel, A. Saffron in phytotherapy: Pharmacology and clinical uses. Wien. Med. Wochenschr. 2007, 157, 315. [CrossRef]

9. Arasteh, A.; Aliyev, A.; Khamnei, S.; Delazar, A.; Mesgari, M.; Mehmannavaz, Y. Crocus sativus on serum glucose, insulin and cholesterol levels in healthy male rats. J. Med. Plants Res. 2010, 4, 397-402.

10. Shirali, S.; Zahra Bathaie, S.; Nakhjavani, M. Effect of Crocin on the Insulin Resistance and Lipid Profile of Streptozotocin-Induced Diabetic Rats. Phytother. Res. 2013, 27, 1042-1047. [CrossRef]

11. Rajaei, Z.; Hadjzadeh, M.-A.-R.; Nemati, H.; Hosseini, M.; Ahmadi, M.; Shafiee, S. Antihyperglycemic and Antioxidant Activity of Crocin in Streptozotocin-Induced Diabetic Rats. J. Med. Food 2013, 16, 206-210. [CrossRef]

12. Asri-Rezaei, S.; Tamaddonfard, E.; Ghasemsoltani-Momtaz, B.; Erfanparast, A.; Gholamalipour, S. Effects of crocin and zinc chloride on blood levels of zinc and metabolic and oxidative parameters in streptozotocin-induced diabetic rats. Avicenna J. Phytomed. 2015, 5, 403-412. [PubMed]

13. Kianbakht, S.; Hajiaghaee, R. Anti-hyperglycemic Effects of Saffron and its Active Constituents, Crocin and Safranal, in Alloxan-Induced Diabetic Rats. J. Med. Plants 2011, 3, 82-89.

14. Mohajeri, D.; Mousavi, G.; Doustar, Y. Antihyperglycemic and pancreas-protective effects of Crocus sativus L. (saffron) stigma-ethanolic extract on rats with alloxan-induced diabetes. Sci. Alert 2009, 9, 302-310. [CrossRef] 
15. Kang, C.; Lee, H.; Jung, E.-S.; Seyedian, R.; Jo, M.; Kim, J.; Kim, J.-S.; Kim, E. Saffron (Crocus sativus L.) increases glucose uptake and insulin sensitivity in muscle cells via multipathway mechanisms. Food Chem. 2012, 135, 2350-2358. [CrossRef] [PubMed]

16. Maeda, A.; Kai, K.; Ishii, M.; Ishii, T.; Akagawa, M. Safranal, a novel protein tyrosine phosphatase 1B inhibitor, activates insulin signaling in C2C12 myotubes and improves glucose tolerance in diabetic KK-Ay mice. Mol. Nutr. Food Res. 2014, 58, 1177-1189. [CrossRef] [PubMed]

17. Xi, L.; Qian, Z.; Xu, G.; Zheng, S.; Sun, S.; Wen, N.; Sheng, L.; Shi, Y.; Zhang, Y. Beneficial impact of crocetin, a carotenoid from saffron, on insulin sensitivity in fructose-fed rats. J. Nutr. Biochem. 2007, 18, 64-72. [CrossRef]

18. Lee, I.-A.; Lee, J.H.; Baek, N.-I.; Kim, D.-H. Antihyperlipidemic Effect of Crocin Isolated from the Fructus of Gardenia jasminoides and Its Metabolite Crocetin. Biol. Pharm. Bull. 2005, 28, 2106-2110. [CrossRef]

19. Sheng, L.; Qian, Z.; Zheng, S.; Xi, L. Mechanism of hypolipidemic effect of crocin in rats: Crocin inhibits pancreatic lipase. Eur. J. Pharmacol. 2006, 543, 116-122. [CrossRef]

20. Xu, G.-L.; Yu, S.-Q.; Gong, Z.-N.; Zhang, S.-Q. Study of the effect of crocin on rat experimental hyperlipemia and the underlying mechanisms. Zhongguo Zhong Yao Za Zhi 2005, 30, 369-372.

21. Lee, I.-A.; Min, S.-W.; Kim, D. Lactic acid bacteria increases hypolipidemic effect of crocin isolated from fructus of Gardenia jasminoides. J. Microbiol. Biotechnol. 2006, 16, 1084-1089.

22. Mashmoul, M.; Azlan, A.; Yusof, B.N.M.; Khaza'ai, H.; Mohtarrudin, N.; Boroushaki, M.T. Effects of saffron extract and crocin on anthropometrical, nutritional and lipid profile parameters of rats fed a high fat diet. J. Funct. Foods 2014, 8, 180-187. [CrossRef]

23. Samarghandian, S.; Azimi-Nezhad, M.; Samini, F. Ameliorative Effect of Saffron Aqueous Extract on Hyperglycemia, Hyperlipidemia, and Oxidative Stress on Diabetic Encephalopathy in Streptozotocin Induced Experimental Diabetes Mellitus. BioMed Res. Int. 2014, 2014, 112, 920857. [CrossRef] [PubMed]

24. Abe, K.; Saito, H. Effects of saffron extract and its constituent crocin on learning behaviour and long-term potentiation. Phytother. Res. 2000, 14, 149-152. [CrossRef]

25. Imenshahidi, M.; Razavi, B.M.; Faal, A.; Gholampoor, A.; Mousavi, S.M.; Hosseinzadeh, H. Effects of chronic crocin treatment on desoxycorticosterone acetate (doca)-salt hypertensive rats. Iran. J. Basic Med. Sci. 2014, 17, 9-13.

26. Imenshahidi, M.; Razavi, M.; Faal, A.; Gholampoor, A.; Mousavi, S.; Hosseinzadeh, H. The effect of chronic administration of safranal on systolic blood pressure in rats. Iran. J. Pharm. Res. 2015, 14, 585-590. [CrossRef]

27. Imenshahidi, M.; Razavi, B.M.; Faal, A.; Gholampoor, A.; Mousavi, S.M.; Hosseinzadeh, H. The effect of chronic administration of saffron (Crocus sativus) stigma aqueous extract on systolic blood pressure in rats. Jundishapur J. Nat. Pharm. Prod. 2013, 8, 175-179. [CrossRef]

28. Liu, J.; Qian, Z. Effects of crocin on cholestane-3beta-5alpha-6beta-triol induced apoptosis and related gene expression of cultured endothelial cells. J. China Pharm. Univ. 2005, 36, 254.

29. Xu, G.; Gong, Z.; Yu, W.; Gao, L.; He, S.; Qian, Z. Increased Expression Ratio of Bcl-2/Bax Is Associated with Crocin-Mediated Apoptosis in Bovine Aortic Endothelial Cells. Basic Clin. Pharmacol. Toxicol. 2007, 100, 31-35. [CrossRef]

30. Xu, G.-L.; Qian, Z.-Y.; Yu, S.-Q.; Gong, Z.-N.; Shen, X.-C. Evidence of crocin against endothelial injury induced by hydrogen peroxide in vitro. J. Asian Nat. Prod. Res. 2006, 8, 79-85. [CrossRef]

31. Ma, S.; Liu, B.; Zhou, S.; Xu, X.; Yang, Q.; Zhou, J. Pharmacological studies of glycosides of saffron crocus (Crocus sativus).II.Effects on blood coagulation, platelet aggregation and thromobosis. Zhong Cao Yao 1999, 30, 196-198.

32. He, S.-Y.; Qian, Z.-Y.; Tang, F.-T.; Wen, N.; Xu, G.-L.; Sheng, L. Effect of crocin on experimental atherosclerosis in quails and its mechanisms. Life Sci. 2005, 77, 907-921. [CrossRef] [PubMed]

33. He, S.-Y.; Qian, Z.-Y.; Wen, N.; Tang, F.-T.; Xu, G.-L.; Zhou, C.-H. Influence of crocetin on experimental atherosclerosis in hyperlipidamic-diet quails. Eur. J. Pharmacol. 2007, 554, 191-195. [CrossRef] [PubMed]

34. Khori, V.; Rakhshan, E.; Mirabbas, A. A study of the role of nitric oxide in the mechanism of action of hydroalcoholic extract of saffron (Crocus sativus) on the electrophysiological properties of the rabbit atrioventricular node. In Proceedings of the II International Symposium on Saffron Biology and Technology, Masshad, Iran, 28-30 October 2016; pp. 351-358.

35. Boskabady, M.H.; Shafei, M.N.; Shakiba, A.; Sefidi, H.S. Effect of aqueous-ethanol extract from Crocus sativus (saffron) on guinea-pig isolated heart. Phytother. Res. 2008, 22, 330-334. [CrossRef] [PubMed] 
36. Du, P.; Qian, Z.Y.; Shen, X.C.; Rao, S.Y.; Wen, N. Effectiveness of crocin against myocardial injury. Chin. New Drugs J. 2005, 14, 1424.

37. Zhang, R.; Zhi-Yu, Q.; Xiao-Yuan, H.; Zhen, C.; Jun-Ling, Y.; Hamid, A. Comparison of the effects of crocetin and crocin on myocardial injury in rats. Chin. J. Nat. Med. 2009, 7, 223-227. [CrossRef]

38. Goyal, S.N.; Arora, S.; Sharma, A.K.; Joshi, S.; Ray, R.; Bhatia, J.; Kumari, S.; Arya, D.S. Preventive effect of crocin of Crocus sativus on hemodynamic, biochemical, histopathological and ultrastuctural alterations in isoproterenol-induced cardiotoxicity in rats. Phytomedicine 2010, 17, 227-232. [CrossRef]

39. EElsherbiny, N.M.; Salama, M.F.; Said, E.; El-Sherbiny, M.; Al-Gayyar, M.M. Crocin protects against doxorubicin-induced myocardial toxicity in rats through down-regulation of inflammatory and apoptic pathways. Chem. Biol. Interact. 2016, 247,39-48. [CrossRef]

40. Farshid, A.A.; Tamaddonfard, E.; Moradi-Arzeloo, M.; Mirzakhani, N. The effects of crocin, insulin and their co-administration on the heart function and pathology in streptozotocin-induced diabetic rats. Avicenna J. Phytomed. 2016, 6, 658-670.

41. Feidantsis, K.; Mellidis, K.; Galatou, E.; Sinakos, Z.; Lazou, A. Treatment with crocin improves cardiac dysfunction by normalizing autophagy and inhibiting apoptosis in STZ-induced diabetic cardiomyopathy. Nutr. Metab. Cardiovasc. Dis. 2018, 28, 952-961. [CrossRef]

42. Cai, J.; Yi, F.F.; Bian, Z.Y.; Shen, D.F.; Yang, L.; Yan, L.; Tang, Q.-Z.; Yang, X.-C.; Li, H. Crocetin protects against cardiac hypertrophy by blocking MEK-ERK1/2 signalling pathway. J. Cell. Mol. Med. 2009, 13, 909-925. [CrossRef]

43. Didangelos, T.; Doupis, J.; Veves, A. Oxidative Stress in Diabetes Mellitus and Possible Interventions. In Studies in Diabetes; Obrosova, I., Stevens, M.J., Yorek, M.A., Eds.; Springer New York: New York, NY, USA, 2014; pp. 237-261.

44. Azimi, P.; Ghiasvand, R.; Feizi, A.; Hariri, M.; Abbasi, B. Effects of cinnamon, cardamom, saffron, and ginger consumption on markers of glycemic control, lipid profile, oxidative stress, and inflammation in type 2 diabetes patients. Rev. Diabet. Stud. RDS 2014, 11, 258-266. [CrossRef] [PubMed]

45. Azimi, P.; Ghiasvand, R.; Feizi, A.; Hosseinzadeh, J.; Bahreynian, M.; Hariri, M.; Khosravi-Boroujeni, H. Effect of cinnamon, cardamom, saffron and ginger consumption on blood pressure and a marker of endothelial function in patients with type 2 diabetes mellitus: A randomized controlled clinical trial. Blood Press. 2016, 25, 133-140. [CrossRef] [PubMed]

46. Ebrahimi, F.; Aryaeian, N.; Pahlavani, N.; Abbasi, D.; Hosseini, A.F.; Fallah, S.; Moradi, N.; Heydari, I. The effect of saffron (Crocus sativus L.) supplementation on blood pressure, and renal and liver function in patients with type 2 diabetes mellitus: A double-blinded, randomized clinical trial. Avicenna J. Phytomed. 2019, 9, 322-333. [PubMed]

47. Ebrahimi, F.; Sahebkar, A.; Aryaeian, N.; Pahlavani, N.; Fallah, S.; Moradi, N.; Abbas, D.; Hosseini, A.F. Effects of saffron supplementation on inflammation and metabolic responses in type 2 diabetic patients: A randomized, double-blind, placebo-controlled trial. Diabetes Metab. Syndr. Obes. Targets Ther. 2019, 12, 2107-2115. [CrossRef]

48. Milajerdi, A.; Jazayeri, S.; Bitarafan, V.; Hashemzadeh, N.; Shirzadi, E.; Derakhshan, Z.; Mahmoodi, M.; Rayati, A.; Djazayeri, A.; Akhondzadeh, S. The effect of saffron (Crocus sativus L.) hydro-alcoholic extract on liver and renal functions in type 2 diabetic patients: A double-blinded randomized and placebo control trial. J. Nutr. Intermed. Metab. 2017, 9, 6-11. [CrossRef]

49. Milajerdi, A.; Jazayeri, S.; Hashemzadeh, N.; Shirzadi, E.; Derakhshan, Z.; Djazayeri, A.; Akhondzadeh, S. The effect of saffron (Crocus sativus L.) hydroalcoholic extract on metabolic control in type 2 diabetes mellitus: A triple-blinded randomized clinical trial. J. Res. Med Sci. 2018, 23, 16. [CrossRef]

50. Moravej Aleali, A.; Amani, R.; Shahbazian, H.; Namjooyan, F.; Latifi, S.M.; Cheraghian, B. The effect of hydroalcoholic Saffron (Crocus sativus L.) extract on fasting plasma glucose, HbA1c, lipid profile, liver, and renal function tests in patients with type 2 diabetes mellitus: A randomized double-blind clinical trial. Phytother. Res. 2019, 33, 1648-1657. [CrossRef]

51. Sepahi, S.; Mohajeri, S.A.; Hosseini, S.M.; Khodaverdi, E.; Shoeibi, N.; Namdari, M.; Tabassi SA, S. Effects of Crocin on diabetic maculopathy: A placebo-controlled randomized clinical trial. Am. J. Ophthalmol. 2018, 190, 89-98. [CrossRef] 
52. Javandoost, A.; Afshari, A.; Nikbakht-Jam, I.; Khademi, M.; Eslami, S.; Nosrati, M.; Foroutan-Tanha, M.; Sahebkar, A.; Tavalaie, S.; Ghayour-Mobarhan, M.; et al. Effect of crocin, a carotenoid from saffron, on plasma cholesteryl ester transfer protein and lipid profile in subjects with metabolic syndrome: A double blind randomized clinical trial. ARYA Atheroscler. 2017, 13, 245-252.

53. Kermani, T.; Kazemi, T.; Molki, S.; Ilkhani, K.; Sharifzadeh, G.; Rajabi, O. The efficacy of crocin of saffron (Crocus sativus L.) on the components of metabolic syndrome: A randomized controlled clinical trial. J. Res. Pharm. Pract. 2017, 6, 228-232.

54. Nikbakht-Jam, I.; Khademi, M.; Nosrati, M.; Eslami, S.; Foroutan-Tanha, M.; Sahebkar, A.; Tavalaie, S.; Ghayour-Mobarhan, M.; Ferns, G.A.A.; Hadizadeh, F.; et al. Effect of crocin extracted from saffron on pro-oxidant-anti-oxidant balance in subjects with metabolic syndrome: A randomized, placebo-controlled clinical trial. Eur. J. Integr. Med. 2016, 8, 307-312. [CrossRef]

55. Zilaee, M.; Soukhtanloo, M.; Ghayour-Mobarhan, M.; Shemshian, M.; Salehi, M.; Ferns, G.A. Effect of saffron on serum leptin levels in patients with metabolic syndrome, a double-blind, randomized and placebo-controlled trial study. Prog. Nutr. 2018, 20, 140-144.

56. Karimi-Nazari, E.; Nadjarzadeh, A.; Masoumi, R.; Marzban, A.; Mohajeri, S.A.; Ramezani-Jolfaie, N.; Salehi-Abargouei, A. Effect of saffron (Crocus sativus L.) on lipid profile, glycemic indices and antioxidant status among overweight/obese prediabetic individuals: A double-blinded, randomized controlled trial. Clin. Nutr. ESPEN 2019, 34, 130-136. [CrossRef] [PubMed]

57. Abedimanesh, N.; Bathaie, S.Z.; Abedimanesh, S.; Motlagh, B.; Separham, A.; Ostadrahimi, A. Saffron and crocin improved appetite, dietary intakes and body composition in patients with coronary artery disease. J. Cardiovasc. Thorac. Res. 2017, 9, 200-208. [CrossRef]

58. Gout, B.; Bourges, C.; Paineau-Dubreuil, S. Satiereal, a Crocus sativus L. extract, reduces snacking and increases satiety in a randomized placebo-controlled study of mildly overweight, healthy women. Nutr. Res. 2010, 30, 305-313. [CrossRef]

59. Pourmasoumi, M.; Hadi, A.; Najafgholizadeh, A.; Kafeshani, M.; Sahebkar, A. Clinical evidence on the effects of saffron (Crocus sativus L.) on cardiovascular risk factors: A systematic review meta-analysis. Pharmacol. Res. 2019, 139, 348-359. [CrossRef]

60. Alavizadeh, S.H.; Hosseinzadeh, H. Bioactivity assessment and toxicity of crocin: A comprehensive review. Food Chem. Toxicol. 2014, 64, 65-80. [CrossRef]

61. Razavi, B.M.; Hosseinzadeh, H. Saffron: A promising natural medicine in the treatment of metabolic syndrome. J. Sci. Food Agric. 2017, 97, 1679-1685. [CrossRef]

62. Tome-Carneiro, J.; Visioli, F. Polyphenol-based nutraceuticals for the prevention and treatment of cardiovascular disease: Review of human evidence. Phytomedicine 2016, 23, 1145-1174. [CrossRef]

63. Bjelakovic, G.; Nikolova, D.; Gluud, L.L.; Simonetti, R.G.; Gluud, C. Mortality in randomized trials of antioxidant supplements for primary and secondary prevention: Systematic review and meta-analysis. JAMA 2007, 297, 842-857. [CrossRef]

64. Moyer, M.W. The myth of antioxidants. Sci. Am. 2013, 308, 62-67. [CrossRef] [PubMed]

65. Yabe, D.; Seino, Y.; Fukushima, M.; Seino, S. $\beta$ cell dysfunction versus insulin resistance in the pathogenesis of type 2 diabetes in East Asians. Curr. Diabetes Rep. 2015, 15, 36. [CrossRef] [PubMed]

(C) 2020 by the authors. Licensee MDPI, Basel, Switzerland. This article is an open access article distributed under the terms and conditions of the Creative Commons Attribution (CC BY) license (http://creativecommons.org/licenses/by/4.0/). 